\title{
Effect of Stocking Density, Dietary Vitamin D3 and Probiotic Supplementation on Carcass Traits and Blood Parameters of Broiler Chickens
}

\author{
S. A. El-Shony ${ }^{1}$, G. M. El-Gendi ${ }^{1}$, H. R. Samak ${ }^{2}$, and O. H. El-Garhy ${ }^{1}$ \\ ${ }^{1}$ Animal production Dep., Fac. of Agric., Benha University., Egypt \\ ${ }^{2}$ Animal Production Research Institute, Ministry of Agriculture, Egypt \\ Corresponding author: osama.elsayed1977@yahoo.com
}

\begin{abstract}
The present study was conducted to evaluate the effect of stocking density, dietary supplementation of probiotic and Vitamin D3 on carcass yield and blood biochemistry of broiler chickens. A total number of 360 unsexed broiler chicks (Evian), one-day old, nearly similar in live body weight were used in this study. Chicks were randomly divided into equal 12 treatments in a $2 \mathrm{X} 3 \mathrm{X} 2$ factorial arrangement experiment. Birds were allocated in two main experimental groups (each of 180 birds), the first main group kept under normal stocking density (LSD) with 10 birds $/ \mathrm{m}^{2}$ and the second main group kept under high stocking density (HSD) with16 birds $/ \mathrm{m}^{2}$. Each main group was divided into three groups (each of 60 birds) fed on diets supplemented with probiotic at a levels of 0,1 and $2 \mathrm{~g} / \mathrm{kg}$ diet, respectively. Each group divided into two sub-group (each of 30 birds) and supplemented with fed vit.-D3 at a level of 250 and $500 \mathrm{IU} / \mathrm{kg}$ diet, respectively. Results obtained showed that raised broiler chickens under LSD had significantly improved absolute and relative carcass, giblets and total edible weight Moreover, it has significantly increased plasma total protein, albumin (A), globulin (G), cholesterol, triglyceride and it has decreased plasma transaminase enzymes of AST and ALT, compared with those rose under HSD. Broiler chicks fed on diet supplemented with probiotic at level of $2 \mathrm{~g} / \mathrm{kg}$ diet showed higher plasma total protein, albumin and A/G ratio, cholesterol and triglycerides than those fed on the other levels of probiotic supplementations. While, it has recorded lowest plasma AST and ALT levels, compared with the other experimental groups. Feeding broiler chickens on high level of Vit.-D3 (500 IU/ Kg diet) has significantly increased plasma protein fractions and decreased plasma AST and ALT levels, compared with those fed diet supplemented with low level of Vit.-D3 (250 IU/Kg) that caused significant decreased plasma cholesterol and triglycerides. Finally, it could be recommended to apply LSD (10 birds/ $\left.\mathrm{m}^{2}\right)$, high level of Vit.D3 $(500 \mathrm{IU} / \mathrm{Kg}$ ) and probiotic at a level of $2 \mathrm{~g} / \mathrm{kg}$ diet to improve carcass yield and blood biochemistry of broiler chickens.
\end{abstract}

Key words: broilers, stocking density, probiotic, Vit.-D3, carcass and blood parameters

\section{Introduction}

The performance of broilers is governed by different factors like environment, nutrition, management, and genetic makeup. Optimal environment and nutrition must be provided for better performance of broilers. High stocking density (HSD) has been reported for its beneficial economical impact if it remains within optimum range (Dozier et al., 2006). HSD reduces the fixed cost of production and produces more kilograms of chicken per area up to a certain extent with increasing profitability (Puron et al., 1995). A higher profitability per kilogram chicken can be obtained by increasing the stocking density if the performance of birds remains constant (Feddes et al., 2002). However, it seems to be impractical due to the fact that the health and performance of broilers is compromised if the placement density goes beyond 34 to $40 \mathrm{~kg} / \mathrm{m}^{2}$ that assuming to be $2.5 \mathrm{~kg}$ final body weight $(\mathrm{BW})$ on an average at the end of growing period (Estevez, 2007). High stocking density (HSD) reduces the performance of broilers due to a number of factors. It accounts for increased ammonia and reduced access to feed and water in addition to poor air quality because of insufficient air exchange at the level of bird. Consequently, a high environmental temperature is observed in the microclimate of the bird resulting in a reduced dissipation of body heat to the environment (Feddes et al., 2002), thus, the ability of the farmers to keep the balance between HSD and the confortable environmental management conditions in the farm, it may be the main key to increase the production performance of broiler chicken under HSD condition. High stoking density poses a stress on the broilers and it is believed that stressful conditions such as heat stress and crowding disrupt the microbial ecology of the bird's intestine, thereby causing dysbiosis(Guardia et al., 2011). One of the possible approaches employed to maintain the performance, intestinal health, humeral immunity, and to avoid physiological stress in broiler exposed to heat stress is the use of dietary probiotics (Sohail et al., 2012) which enhance the performance without being digested. Direct feed microbial, known as probiotics, can improve the performance (Huang et al., 2004), nutrient digestibility (Li et al. 2008), immunity (Teo and Tan, 2007), gut microflora ecology (Yu et al., 2008), and inhibition of pathogens (Mountzouris et al., 2009) in birds. In poultry feeding, cholecalciferol (VIT-D3) is necessary for the intestinal absorption, blood transport and efficient metabolism of calcium and phosphorus. In addition, the VIT-D3 has an 
important role for in the development of skin and blood cells, as well it is involved in controlling cellular activities of host immune system (Lymboussaki et al., 2009).Vitamin D is an essential metabolite for bone formation and maintenance in all vertebrate animals primarily because cholecalciferol (D3) controls calcium (Ca) and phosphorus (P) homeostasis within the vertebrate body (Hurwitz $\boldsymbol{e t}$ al. 1984).The $D 3$, the first metabolite of vitamin $D$ is transferred from the skin and gastrointestinal tract via serum to the liver where it undergoes the first hydroxylation to 25 hydroxy cholecaciferol [25(OH)D3]. For 25(OH)D3, the second metabolite of vitamin $\mathrm{D}$ is the storage form of vitamin $\mathrm{D}$. Thus25(OH)D3 remains in serum until it is further hydroxylated in the kidney to become the active hormonal metabolite of vitamin D known as 1,25dihydroxy cholecalciferol [1,25-(OH)2D3] (Fraser and Kodicek (1970). Until recently, tibial dyschondroplasia was the main leg problem affecting broilers (Almeida Paz et al., 2008), specially under the HSD during the growing period of broiler chickens. Vitamin $\mathrm{D}$ is added to broiler diets to provide $\mathrm{Ca}$ balance, and to improve $\mathrm{Ca}$ intestinal absorption (Waldenstedt, 2006). The aim of this study was to evaluate the effect of stoking density, dietary supplementation of probiotic and Vitamin D3 on carcass yield and some blood biochemical parameters of broiler chickens.

\section{Material and Methods:}

\section{Experimental design:}

A total number of 360 unsexed broiler chicks (Evian), one-day old, nearly a similar live body weight were used in this study. Chicks were randomly divided into equal 12 treatments in a 2X3X2 factorial arrangement experiment. Birds were allocated in two main experimental groups (each of 180 birds), the first main group kept under normal stocking density (SD) with $10 \mathrm{birds} / \mathrm{m}^{2}$ and the second main group kept under high stocking density (HSD) with16 birds $/ \mathrm{m}^{2}$. Each main group was divided into three groups (each of 60 birds) fed on diets supplemented with probiotic at levels of 0,1 and $2 \mathrm{~g} / \mathrm{kg}$ diet, respectively. Each group divided into two sub-groups (each of 30 birds) and supplemented with fed Vit.-D3at levels of 250 and $500 \mathrm{IU} / \mathrm{kg}$ diet, respectively. Chicks were kept under similar, standard hygienic and environmental conditions. Wood shaving was used at $10 \mathrm{~cm}$ depth as a litter and the wetting litter was continually changed by a fresh one. Floor brooders with gas heaters were used for brooding chicks. Brooding temperature was maintained at $35^{\circ} \mathrm{C}$ during the first 5 days of chick's age, and then decreased by $2^{\circ} \mathrm{C}$ weekly until the end of the $4^{\text {th }}$ week. The lighting program was $24 \mathrm{~h}$ light during the first 5 days of age, then it decreased from 6 to 42 days of age (the end of the experiment) to 23 hours light and 1 hour dark was applied. Feed and water were offered ad-libitum. Chicks were fed starter and grower diets. The basal diet was formulated according to the recommended requirements of NRC (1994) as showed in table (1):

Table 1. The composition and calculated analyses of experimental starter and grower diets:

\begin{tabular}{lcc}
\hline Ingredients \% & $\begin{array}{c}\text { Starter } \\
(\mathbf{0 - 4}) \mathbf{w k s}\end{array}$ & $\begin{array}{c}\text { Grower } \\
\mathbf{( 4 - 6 )} \mathbf{w k s}\end{array}$ \\
\hline Yellow corn $(8.5 \%)$ & 61.00 & 66.00 \\
Soybean meal (44\% CP) & 35.50 & 26.00 \\
Corn gluten meal (60\% CP) & 0.00 & 1.00 \\
Vegetable oil & 0.00 & 3.00 \\
Di. calcium phosphate & 1.70 & 1.70 \\
Calcium carbonate & 1.10 & 1.20 \\
Sodium chloride & 0.30 & 0.30 \\
Vit. and M.n. permix* & 0.30 & 030 \\
DL. Methionine 99\% & 0.10 & 0.16 \\
L. Lysine hydrochloride $78 \%$ & 0.00 & 0.04 \\
Total calculated analysis & 100 & 100 \\
ME. Kcal / Kg) & 3135 & 2839 \\
Crude protein & 20.86 & 17.81 \\
Crude fat & 2.67 & 5.80 \\
Crude fiber & 2.73 & 2.49 \\
Calcium & 0.93 & 0.95 \\
Available P & 0.43 & 0.42 \\
Methionine & 0.46 & 0.47 \\
Methionine + Cystine & 0.83 & 0.79 \\
Lysine & 1.21 & 0.99 \\
\hline Caculat
\end{tabular}

Calculated analysis \%**

*Each 3.0 Kg of the Vit. and Min . premix contains : 
Vit.A, 12000000 IU; Vit.E, 10 g; Vit.K3, 2.0 g; Vit.B1; 1.0 g; Vit.B2, 5 g; Vit. B6;1.5 g; Vit. B12, 10 mg; choline choloride, 250 g; Biotn, 50 mg; folic acid,1 g; nicotinic acid, 30 g; Ca Pantothenate, 10 g; Zn, 50 g; Cu, 10 g; Fe, 30 g; Co,100 mg; Se, $100 \mathrm{mg} ; \mathrm{I}, 1 \mathrm{~g}$; Mn, $60 \mathrm{~g}$ and antioxidant, $10 \mathrm{~g}$ and complete to $3.0 \mathrm{~kg}$ by calcium carbonate.

\section{Parameters estimation and data collection: Slaughtering and carcass characteristics:}

Carcass characteristic for random sample of 3 birds from each treatment were performed at the end of the experimental period (6 weeks). Birds' chosen were deprived from feed for 16 hours before slaughtering after which they were individually weighed to the nearest $g$ and killed by cutting the throat and the jugular veins with a sharp knife near the first neck vertebra. Birds were individually reweighed after complete bleeding. Shank and head were separated, the birds were then eviscerated and intestine, gizzard, lungs, spleen, liver, heart and all internal organs were removed. The carcass and giblets (empty gizzard, liver and heart) were separately weighed. The proportional weights to live body weight of giblets, carcass and total edible parts were calculated as follows:

$$
\begin{aligned}
& \text { Giblets weight }(\boldsymbol{\%})=\frac{\mathbf{G W}}{\mathbf{L W}} \times \mathbf{1 0 0} \\
& \text { Edible parts }(\boldsymbol{\%})=\frac{\mathbf{E W}+\mathbf{G W}}{\mathbf{L W}} \times \mathbf{1 0 0} \\
& \begin{array}{ll}
\text { Whereas: } \quad \mathrm{LW} & =\text { live body weight } \\
\mathrm{EW} & =\text { eviscerated weight } \\
\mathrm{GW} & =\text { giblets weight }
\end{array}
\end{aligned}
$$

Blood samples for chemical analysis were individually obtained from 5 birds randomly chosen from each treatment at 4 and 6 weeks of birds' age from the wing vein. Heparinized blood samples were centrifuged at $2500 \mathrm{rpm}$ for 15 -min. Plasma samples were then stored in the deep freezer at approximately $-20 \pm 1^{\circ} \mathrm{C}$ until the time of chemical analysis. Plasma total protein, albumin, globulin, $\mathrm{A} / \mathrm{G}$ ratio, triglycerides, cholesterol, creatinine, uric acid, calcium, inorganic phosphorus, asparatate aminotransferase (AST) and glutamic oxaloacetic transaminase(ALT) were calorimetrically determined using commercial kits.

\section{Statistical analysis}

Analysis of variance was calculated using SAS procedure guide (SAS 2004) using the following linear model:

$$
\begin{aligned}
& \mathbf{X}_{i j h k}=\boldsymbol{\mu}+S_{i}+P_{j}+V_{h}+S_{i j}+S V_{i h}+P V_{j h}+S P V_{i j h}+ \\
& E_{i j h k} \\
& \text { Whereas: } \\
& X_{i j h k}=\text { the } k^{\text {th }} \text { observation } \\
& \mu=\text { overall mean } \\
& S_{i}=\text { the effect of } i^{\text {th }} \text { stocking density } \\
& P_{j}=\text { the effect of } j^{\text {th }} \text { level of probiotic } \\
& V_{h}=\text { the effect of } h^{\text {th }} \text { level of vitamin D3 } \\
& S P_{i j}=\text { the effect of the interaction between the } i^{\text {th }} \\
& \quad \text { stocking density and the } j^{\text {th }} \text { probiotic. }
\end{aligned}
$$

$\mathrm{SV}_{\mathrm{ih}}=$ the effect of the interaction between the $\mathrm{i}^{\text {th }}$ stocking density and the $\mathrm{h}^{\text {th }}$ vitamin D3.

$\mathrm{PV}_{\mathrm{jh}}=$ the effect of the interaction between the $\mathrm{j}^{\text {th }}$ probiotic and the $\mathrm{h}^{\text {th }}$ vitamin D3.

$S P V_{i j h}=$ the effect of the interaction between the $i^{\text {th }}$ stocking density, the $\mathrm{j}^{\text {th }}$ levels of probiotic and the $\mathrm{h}^{\text {th }}$ levels of vitamin D3

$\mathrm{E}_{\mathrm{ijhk}}=$ the experimental error

Significant differences among group were tested using Duncan multiple range test

(Duncan. 1955).

\section{Results and Discussion}

\section{Carcass traits:}

Results obtained in table (2) showed highly significant variations were found in absolute weights of carcass, giblets and total edible parts due to the effect of stocking density (SD) of birds. While, no significant variations were found in absolute weights of carcass, giblets and total edible parts due to either probiotic $(\mathrm{P})$ or Vitamin-D3 (V.D3) supplementation levels. However, significant variations were found in all carcass traits, except proportional weights of carcass and total edible parts of broiler chickens due to effect of the interaction between SD X V.D3 X P. Broiler chickens kept under LSD (10 bird $/ \mathrm{m}^{2}$ ) recorded the highest absolute weights of carcass, giblets and total edible parts (1806.1, 100.3 and $1906.4 \mathrm{~g}$ respectively), compared with those kept under HSD (16 bird/ $\mathrm{m}^{2}$ ) which recorded the lowest absolute weights of carcass, giblets and total edible parts $(1475,3,83.6$ and $1558.9 \mathrm{~g}$ respectively). These results agreed with those concluded by Adeyemo et al. (2016) who found that broiler chickens raised in high stocking density up to stocking density 14 birds $/ \mathrm{m}^{2}$ showed significant improve of carcass characteristics. However, it is disagree with those reported by Rudzani et. al., (2017) and Goo et al., (2019) they found no significant $(\mathrm{P}>0.05)$ effect of stocking density on carcass, eviscerated carcass, breast, and abdominal fat yields of broiler chickens.

Although there were no significant effects of Probiotic and Vit.-D3on absolute and relative weights of carcass, giblets and total edible parts, it is clearly observed that chicks fed on diet supplemented with probiotic at a level of $1 \mathrm{~g} / \mathrm{kg}$ diet showed a higher absolute weights of carcass, giblets and total edible parts $(1682.5,93.3$ and $1775.8 \mathrm{~g}$, respectively) than those fed on probiotic at a level of $2 \mathrm{~g} / \mathrm{kg}$ diet and those fed diet without probiotic. Moreover, chicks that fed on diet supplemented with V.D3 at a level of $500 \mathrm{IU} / \mathrm{Kg}$ diet showed higher averages of absolute weights of carcass, giblets and total edible parts than those fed diet supplemented with V.D3 at a level of $250 \mathrm{IU} / \mathrm{Kg}$ diet. The interaction between LSD X P2 X V2 showed significantly the higher averages of absolute and relative weights of carcass and total edible parts. While the interaction between LSD X P0 X V2 showed 
significantly the higher averages of absolute and relative weights of giblets when compared with

different interactions applied.

Table 2. Least -square means and pooled standard error for carcass characteristics of different experimental groups as affected by studied factors

\begin{tabular}{|c|c|c|c|c|c|c|c|}
\hline \multirow{3}{*}{ Treatments } & \multirow{3}{*}{ level } & \multicolumn{6}{|c|}{ Carcass traits } \\
\hline & & \multicolumn{2}{|c|}{ Carcass weight } & \multicolumn{2}{|c|}{ Giblets weight } & \multicolumn{2}{|c|}{ Total edible weight } \\
\hline & & $\mathbf{g}$ & $\%$ & $\mathbf{g}$ & $\%$ & $\mathbf{g}$ & $\%$ \\
\hline Stoking density(SD) & 10 & $1806.11^{\mathrm{a}}$ & 74.39 & $100.27^{\mathrm{a}}$ & 4.13 & $1906.38^{\mathrm{a}}$ & 74.53 \\
\hline bird $/ \mathrm{m}^{2}$ & 16 & $1475.27^{b}$ & 73.63 & $83.61^{\mathrm{b}}$ & 4.16 & $1558.88^{\mathrm{b}}$ & 75.82 \\
\hline MSE & & 18.40 & 0.36 & 1.51 & 0.07 & 18.30 & 0.38 \\
\hline Probiotic (P) & 0 & 1570.0 & 73.24 & 91.08 & 4.30 & 1662.08 & 77.54 \\
\hline ProDiotic (F) & 1 & 1682.50 & 74.32 & 93.33 & 4.10 & 1775.83 & 78.50 \\
\hline g / kg & 2 & 1669.58 & 74.44 & 92.41 & 4.05 & 1760.0 & 78.48 \\
\hline MSE & & 22.22 & 0.44 & 1.85 & 0.09 & 22.41 & 0.46 \\
\hline Vitamin D3 (V.D3 ) & 250 & 1626.11 & 74.22 & 91.38 & 4.17 & 1717.50 & 78.41 \\
\hline IU/kg & 500 & 1655.27 & 73.80 & 92.50 & 4.12 & 1747.77 & 77.94 \\
\hline MSE & & 18.14 & 0.36 & 1.51 & 0.07 & 18.30 & 0.38 \\
\hline \multicolumn{8}{|l|}{ Interaction } \\
\hline $\operatorname{LSDX~P}_{0} \mathrm{X} \mathrm{V}_{1}$ & $10 \times 0 \times 250$ & $1705.00^{\mathrm{bc}}$ & 73.56 & $96.66^{\mathrm{ab}}$ & $4.16^{\mathrm{ab}}$ & $1801.66^{b}$ & 77.73 \\
\hline $\operatorname{LSDX}_{\mathrm{P}_{0}} \mathrm{X} \mathrm{V}_{2}$ & $10 \times 0 \times 500$ & $1663.33 b$ & 73.40 & $101.66^{\mathrm{a}}$ & $4.50^{\mathrm{a}}$ & $1765.0^{\mathrm{ab}}$ & 77.90 \\
\hline LSDX P $P_{1} X V_{1}$ & $10 \times 1 \times 250$ & $1801.66^{\mathrm{ab}}$ & 75.16 & $98.33^{\mathrm{a}}$ & $4.10^{\mathrm{ab}}$ & $1900.0^{\mathrm{a}}$ & 79,26 \\
\hline LSDX P $P_{1} X V_{2}$ & $10 \times 1 \times 500$ & $1891.66^{\mathrm{a}}$ & 74.20 & $96.03^{\mathrm{ab}}$ & $4.26^{\mathrm{ab}}$ & $2000.0^{\mathrm{a}}$ & 78.46 \\
\hline LSDX $P_{2} X V_{1}$ & $10 \times 2 \times 250$ & $1868.33^{\mathrm{ab}}$ & 74.76 & $100.33^{\mathrm{a}}$ & $4.01^{\mathrm{ab}}$ & $1968.33^{\mathrm{a}}$ & 78.76 \\
\hline $\mathbf{L S D X} \mathbf{P}_{2} X \mathbf{V}_{2}$ & $10 \times 2 \times 500$ & $1906.66^{\mathrm{a}}$ & 75.26 & $96.66^{\mathrm{bc}}$ & $3.80^{\mathrm{b}}$ & $2003.33^{\mathrm{a}}$ & 79.06 \\
\hline $\operatorname{HSDX}_{\mathbf{P}} \mathrm{X} \mathrm{V}_{1}$ & $16 \times 0 \times 250$ & $1418.33^{\mathrm{d}}$ & 73.73 & $85.11^{\mathrm{bc}}$ & $4.43^{\mathrm{ab}}$ & $1503.33^{c}$ & 78.16 \\
\hline $\operatorname{HSDX}_{\mathbf{P}} \mathrm{X} \mathrm{V}_{2}$ & $16 \times 0 \times 500$ & $1493.33^{\mathrm{d}}$ & 72.26 & $85.01^{\text {bc }}$ & $4.10^{\mathrm{ab}}$ & $1578.33^{c}$ & 76.36 \\
\hline HSDX P $P_{1}$ X V & $16 \times 1 \times 250$ & $1503.33^{\mathrm{d}}$ & 73.93 & $85.01^{\mathrm{bc}}$ & $4.13^{\mathrm{ab}}$ & $1588.33^{c}$ & 78.13 \\
\hline HSDX $P_{1} \times V_{2}$ & $16 \times 1 \times 500$ & $1533.33^{\mathrm{d}}$ & 74.20 & $81.66^{c}$ & $3.93^{\mathrm{ab}}$ & $1615.0^{\mathrm{c}}$ & 78.17 \\
\hline $\operatorname{HSDX}_{2} \mathrm{X} \mathrm{V}_{1}$ & $16 \times 2 \times 250$ & $1460.33^{\mathrm{d}}$ & 74.1 & $83.33^{\mathrm{c}}$ & $4.23^{\mathrm{ab}}$ & $1543.33^{c}$ & 78.40 \\
\hline HSDX $P_{2} X V_{2}$ & $16 \times 2 \times 500$ & $1443.33^{\mathrm{d}}$ & 73.50 & $81.66^{\mathrm{c}}$ & $4.16^{\mathrm{ab}}$ & $1525.0^{\mathrm{c}}$ & 77.70 \\
\hline MSE & & 43.83 & 0.90 & 3.69 & 0.19 & 44.29 & 0.95 \\
\hline
\end{tabular}

\section{Blood constituents}

\section{Plasma protein fractions:}

The obtained results in table (3) showed that the broiler chicks kept under LSD recorded significantly the higher averages of plasma total protein, albumin and globulin at $4^{\text {th }}$ and $6^{\text {th }}$ week of age, than those kept under HSD. These results are agree with those reported by Tong et al., (2012) who found significant differences in protein fractions $(\mathrm{P}>0.05)$ of plasma broiler chicken due to the effect of stocking density. Concerning to the effect of probiotic on plasma protein fractions, it is clearly observed that chicks fed on diet supplemented with probiotic at a level of $2 \mathrm{~g} / \mathrm{kg}$ diet showed significantly the higher averages of plasma total protein, albumin, globulin and $\mathrm{A} / \mathrm{G}$ ratio at 4 and 6 wks of age, followed by those fed diet witha level of $1 \mathrm{~g} / \mathrm{kg}$ diet or non- supplemented one, respectively. These results agreed with those reported by Mohammedi et al., (2016) who showed that the supplementation of probiotic in broiler diets had positive effect on plasma albumin value of broiler chickens compared with control. However, it was disagree with those reported by Sherif (2009b) who found that plasma blood parameters of broiler chickens weren't affected by adding probiotic (Avian plus) in their diets. Chicks fed diet supplemented with V.D3 at level of $500 \mathrm{IU} / \mathrm{Kg}$ diet showed significantly the higher averages of plasma total protein, albumin and globulin at 4 and 6 wks of bird's age than those fed diet supplemented with V.D3 at a level of $250 \mathrm{IU} / \mathrm{Kg}$ diet. The interactions between LSD X P1 X V2 showed the highest averages of plasma total protein and globulin mounted (3.16 and $1.86 \mathrm{~g} / \mathrm{dI})$, respectively at the $6^{\text {th }}$ week of age. However, the interaction between HSD X P1X V2 showed the highest average of plasma albumin and $\mathrm{A} / \mathrm{G}$ ratio at the $6^{\text {th }}$ week of bird's age when compared with different interaction effects. 
Table 3. Least -square means and pooled standard error for plasma protein fractions (total protein, albumin and globulin and A/G ratio) of different experimental groups as affected by studied factors

\begin{tabular}{|c|c|c|c|c|c|c|c|c|c|}
\hline \multirow{2}{*}{ Treatment } & \multirow{2}{*}{ level } & \multicolumn{2}{|c|}{$\begin{array}{c}\text { Plasma total } \\
\operatorname{protein}(\mathrm{g} / \mathrm{dI}) \text { at }\end{array}$} & \multicolumn{2}{|c|}{$\begin{array}{c}\text { Plasma } \\
\text { albumin }(\mathrm{g} / \mathrm{dI}) \\
\text { at }\end{array}$} & \multicolumn{2}{|c|}{$\begin{array}{l}\text { Plasma globulin } \\
\text { (g/dI) at }\end{array}$} & \multicolumn{2}{|c|}{$\begin{array}{l}\operatorname{Plasma~A/G} \\
\operatorname{ratio}(g / d l) \text { at }\end{array}$} \\
\hline & & 4 wks & $6 \mathrm{wks}$ & 4wks & 6wks & $4 w k s$ & 6wks & 4wks & 6wks \\
\hline \multirow{2}{*}{$\begin{array}{c}\text { Stoking } \\
\operatorname{density}(\mathrm{SD}) \\
\text { bird//m² }\end{array}$} & 10 & 3.0 & $2.74^{\mathrm{a}}$ & $1.20^{\mathrm{a}}$ & $1.46^{\mathrm{a}}$ & $1.53^{\mathrm{a}}$ & $1.74^{\mathrm{a}}$ & 0.78 & 0.72 \\
\hline & 16 & 2.98 & $2.57^{\mathrm{b}}$ & $1.13^{\mathrm{b}}$ & $1.38^{\mathrm{b}}$ & $1.44^{\mathrm{b}}$ & $1.60^{\mathrm{b}}$ & 0.78 & 0.86 \\
\hline \multirow{4}{*}{$\begin{array}{l}\text { Probiotic }(\mathbf{P}) \\
\mathrm{g} / \mathbf{k g}\end{array}$} & & 0.011 & 0.013 & 0.007 & 0.011 & 0.009 & 0.013 & 0.005 & 0.011 \\
\hline & 0 & $2.56^{\mathrm{c}}$ & $2.89^{c}$ & $1.25^{\mathrm{b}}$ & $1.12^{\mathrm{c}}$ & $1.44^{\mathrm{b}}$ & $1.64^{\mathrm{b}}$ & 0.77 & 0.76 \\
\hline & 1 & $2.67^{\mathrm{b}}$ & $3,08^{\mathrm{b}}$ & $1.37^{\mathrm{a}}$ & $1.17^{\mathrm{b}}$ & $1.49^{\mathrm{a}}$ & $1.71^{\mathrm{a}}$ & 0.78 & 0.80 \\
\hline & 2 & $2.74^{\mathrm{a}}$ & $3.11^{\mathrm{a}}$ & $1.53^{\mathrm{a}}$ & $1.21^{\mathrm{a}}$ & $1.53^{\mathrm{a}}$ & $1.73^{\mathrm{a}}$ & 0.79 & 0.81 \\
\hline MSE & & 0.016 & 0.013 & 0.013 & 0.009 & 0.01 & 0.016 & 0.006 & 0.013 \\
\hline \multirow{2}{*}{$\begin{array}{c}\text { Vitamin } \\
\text { D3(V.D }) \\
\text { IU/kg }\end{array}$} & 250 & $2.63^{\mathrm{b}}$ & $2.94^{\mathrm{b}}$ & $1.15^{\mathrm{b}}$ & $1.30^{\mathrm{b}}$ & $1.47^{\mathrm{b}}$ & $1.64^{\mathrm{b}}$ & 0.78 & 0.79 \\
\hline & 500 & $2.68^{\mathrm{a}}$ & $3.04^{\mathrm{a}}$ & $1.18^{\mathrm{a}}$ & $1.34^{\mathrm{a}}$ & $1.50^{\mathrm{a}}$ & $1.70^{\mathrm{a}}$ & 0.78 & 0.79 \\
\hline MSE & & 0.013 & 0.011 & 0.007 & 0.05 & 0.01 & 0.013 & 0.013 & 0.011 \\
\hline \multicolumn{10}{|l|}{ Interaction } \\
\hline $\operatorname{LSDX}_{P_{0} X V_{1}}$ & $10 \times 0 \times 250$ & $2.62^{\mathrm{dc}}$ & $2.81^{\mathrm{e}}$ & $1.13^{\mathrm{dc}}$ & $1.16^{\mathrm{d}}$ & $1.48^{\mathrm{bc}}$ & $1.65^{\mathrm{cd}}$ & 0.76 & 0.71 \\
\hline $\operatorname{LSDX}_{P_{0}} X V_{2}$ & $10 \times 0 \times 500$ & $2.73^{\mathrm{ab}}$ & $2.98 \mathrm{dc}$ & $1.20^{\mathrm{ab}}$ & $1.26^{\mathrm{c}}$ & $1.51^{\mathrm{ab}}$ & $1.71^{\mathrm{bc}}$ & 0.79 & 0.74 \\
\hline $\operatorname{LSDX} P_{1} X V_{1}$ & $10 \times 1 \times 250$ & $2.74^{\mathrm{ab}}$ & $3.03^{b c}$ & $1.20^{\mathrm{ab}}$ & $1.28^{\mathrm{c}}$ & $1.54^{\mathrm{ab}}$ & $1.75^{\mathrm{bc}}$ & 0.77 & 0.73 \\
\hline LSDX $P_{1} X V_{2}$ & $10 \times 1 \times 500$ & $2.76^{\mathrm{a}}$ & $3.16^{\mathrm{a}}$ & $1.22^{\mathrm{ab}}$ & $1.30^{\mathrm{c}}$ & $1.54^{\mathrm{ab}}$ & $1.86^{\mathrm{a}}$ & 0.79 & 0.69 \\
\hline $\operatorname{LSDX}_{P_{2}} X V_{1}$ & $10 \times 2 \times 250$ & $2.78^{\mathrm{a}}$ & $3.00 \mathrm{dc}$ & $1.22^{\mathrm{a}}$ & $1.30^{\mathrm{c}}$ & $1.56^{\mathrm{a}}$ & $1.70^{\mathrm{cd}}$ & 0.78 & 0.76 \\
\hline $\mathbf{L S D X} \mathrm{P}_{2} \mathrm{X} \mathrm{V}_{2}$ & $10 \times 2 \times 500$ & $2.81^{\mathrm{a}}$ & $3.05^{\mathrm{bc}}$ & $1.25^{\mathrm{a}}$ & $1.26^{\mathrm{c}}$ & $1.57^{\mathrm{a}}$ & $1.78^{\mathrm{ab}}$ & 0.80 & 0.70 \\
\hline $\operatorname{HSDX}_{\mathrm{P}_{0}} \mathrm{X} \mathrm{V}_{1}$ & $16 \times 0 \times 250$ & $2.46^{\mathrm{e}}$ & $2.83^{\mathrm{e}}$ & $1.08^{\mathrm{de}}$ & $1.27^{\mathrm{c}}$ & $1.38^{\mathrm{d}}$ & $1.56^{\mathrm{f}}$ & 0.78 & 0.82 \\
\hline $\mathrm{HSDX}_{\mathrm{P}_{0}} \mathrm{X} \mathrm{V}_{2}$ & $16 \times 0 \times 500$ & $2.45^{\mathrm{e}}$ & $2.93^{\mathrm{d}}$ & $1.06^{\mathrm{e}}$ & $1.30^{\mathrm{c}}$ & $1.38^{\mathrm{d}}$ & $1.63^{\mathrm{de}}$ & 0.77 & 0.79 \\
\hline $\mathrm{HSDX}_{\mathrm{P}_{1}} \mathrm{X} \mathrm{V}_{1}$ & $16 \times 1 \times 250$ & $2.56^{\mathrm{d}}$ & $3.01^{\mathrm{dc}}$ & $1.13^{\mathrm{dc}}$ & $1.40^{b}$ & $1.43^{\mathrm{cd}}$ & $1.61^{\mathrm{de}}$ & 0.79 & 0.86 \\
\hline${ }_{H S D X} P_{1} X V_{2}$ & $16 \times 1 \times 500$ & $2.61^{\mathrm{dc}}$ & $3.11^{\mathrm{ab}}$ & $1.13^{\mathrm{dc}}$ & $1.50^{\mathrm{a}}$ & $1.48^{\mathrm{bc}}$ & $1.61^{\mathrm{de}}$ & 0.76 & 0.92 \\
\hline${ }_{H S D X} P_{2} X V_{1}$ & $16 \times 2 \times 250$ & $2.63^{\mathrm{dc}}$ & $2.98^{\mathrm{dc}}$ & $1.16^{\mathrm{bc}}$ & $1.40^{\mathrm{b}}$ & $1.46^{\mathrm{bc}}$ & $1.58^{\mathrm{f}}$ & 0.79 & 0.89 \\
\hline $\mathrm{HSDX}_{\mathrm{P}_{2}} \mathrm{X} \mathrm{V}_{2}$ & $16 \times 2 \times 500$ & $2.73^{\mathrm{ab}}$ & $3.03^{b c}$ & $1.20^{\mathrm{ab}}$ & $1.43^{\mathrm{ab}}$ & 1.52 & $1.60^{\mathrm{ef}}$ & 0.79 & 0.89 \\
\hline MSE & & 0.03 & 0.02 & 0.01 & 0.02 & 0.02 & 0.03 & 0.01 & 0.02 \\
\hline
\end{tabular}

Plasma cholesterol and triglycerides:

Results obtained in table (4) revealed that, broiler chicks that kept under LSD recorded the higher averages of plasma cholesterol and triglycerides at 4 and 6 wks of bird's age, than those kept under HSD. This result disagree with those reported by Thaxton et al. (2006)who found that stocking density didn't result in a recognizable trend in plasma cholesterol of broiler chickens. Concerning to the effect of probiotic on plasma cholesterol, it is clearly observed that chicks fed on diet supplemented with probiotic at a level of $2 \mathrm{~g} / \mathrm{kg}$ diet showed a higher average of plasma cholesterol at the $6^{\text {th }}$ week $(157.5 \mathrm{~g} / \mathrm{dI})$ of age, followed by those fed diet supplemented with $\lg (156.4 \mathrm{~g} / \mathrm{dI})$, compared with those fed diet without probiotic supplementation (155.0 $\mathrm{g} / \mathrm{dI})$.Opposite results were observed in plasma triglycerides which decreased with increasing dietary levels of probiotic supplementation. These results disagree with those stated by Ignatova et al. (2009) they found that probiotic supplementation leads to a significant decrease in blood plasma cholesterol and/or triglycerides of broiler chickens. Chicks fed diet supplemented with V.D3 at a level of $250 \mathrm{IU} / \mathrm{Kg}$ diet showed the lowest averages of plasma cholesterol $(154.3 \mathrm{~g} / \mathrm{dI})$ and triglycerides $(148.6 \mathrm{~g} / \mathrm{dI})$ at the 6 wks of age when compared with those fed diet supplemented with V.D3 at a level of $500 \mathrm{IU} / \mathrm{Kg}$ (158.3 and $148.9 \mathrm{~g} / \mathrm{dI}$ ) for plasma cholesterol and triglycerides, respectively. The interaction between HSD X P0 XV1 showed the lowest level of plasma cholesterol at the $6^{\text {th }}$ wk of bird's age. However, the interaction effect between LSD X P1 X V1 showed the lowest averages of plasma triglycerides at the 6 wk of bird's age, compared with different interaction effects. 
Table 4. Least - square means and pooled standard error for plasma cholesterol and triglycerides of different experimental groups as affected by studied factors

\begin{tabular}{|c|c|c|c|c|c|}
\hline \multirow[t]{2}{*}{ Treatment } & \multirow[t]{2}{*}{ level } & \multicolumn{2}{|c|}{ Plasma cholesterol (g/dI)at } & \multicolumn{2}{|c|}{$\begin{array}{c}\text { Plasma triglycerides } \\
(\mathrm{g} / \mathrm{dI}) \mathrm{at}\end{array}$} \\
\hline & & $4 w k s$ & 6wks & 4wks & 6wks \\
\hline Stoking density (SD) & 10 & $151.61^{\mathrm{a}}$ & $157.72^{\mathrm{a}}$ & 113.7 & 149.16 \\
\hline bird $/ \mathbf{m}^{2}$ & 16 & $144.44^{\mathrm{b}}$ & $154.88^{b}$ & 113.2 & 148.33 \\
\hline MSE & & 0.38 & 0.49 & 1.12 & 4.45 \\
\hline & 0 & $146.91^{b}$ & $155.00^{\mathrm{b}}$ & 113.66 & 149.50 \\
\hline Probiotic (P) & 1 & $148.33^{\mathrm{a}}$ & $156.41^{\mathrm{ab}}$ & 113.25 & 147.91 \\
\hline g / / g & 2 & $148.80^{\mathrm{a}}$ & $157.50^{\mathrm{a}}$ & 113.58 & 148.83 \\
\hline MSE & & 0.46 & 0.60 & 1.37 & 4.45 \\
\hline 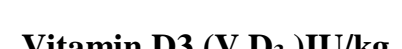 & 250 & $146.05^{\mathrm{b}}$ & $154.27^{b}$ & 113.94 & 148.55 \\
\hline Vitamın $03\left(V . D_{3}\right) 1 \mathrm{U} / \mathrm{kg}$ & 500 & $150.00^{\mathrm{a}}$ & $158.33^{\mathrm{a}}$ & 113.05 & 148.94 \\
\hline MSE & & 0.38 & 0.49 & 1.12 & 4.45 \\
\hline \multicolumn{6}{|l|}{ Interaction } \\
\hline $\operatorname{LSDX}_{0} \times V_{1}$ & $10 \times 0 \times 250$ & $148.00^{\mathrm{de}}$ & $156.33^{\mathrm{cd}}$ & 113.16 & 148.36 \\
\hline $\operatorname{LSDX} \mathrm{P}_{0} \times \mathbf{V}_{2}$ & $10 \times 0 \times 500$ & $152.33^{\mathrm{bc}}$ & $157.00^{\mathrm{cd}}$ & 113.24 & 148.33 \\
\hline LSDX $P_{1} X V_{1}$ & $10 \times 1 \times 250$ & $149.66^{\mathrm{dc}}$ & $155.00^{\mathrm{cd}}$ & 113.12 & 147.45 \\
\hline $\operatorname{LSDX} \mathbf{P}_{1} \times \mathbf{V}_{2}$ & $10 \times 1 \times 500$ & $154.66^{\mathrm{ab}}$ & $159.33^{\mathrm{ab}}$ & 113.62 & 149.53 \\
\hline $\operatorname{LSDX} \mathbf{P}_{2} \mathrm{X} \mathrm{V}_{1}$ & $10 \times 2 \times 250$ & $149.66^{\mathrm{dc}}$ & $156.00^{\mathrm{cd}}$ & 113.35 & 147.90 \\
\hline $\operatorname{LSDX}_{\mathbf{P}} \mathrm{X} \mathrm{V}_{2}$ & $10 \times 2 \times 500$ & $155.33^{\mathrm{a}}$ & $162.66^{\mathrm{a}}$ & 113.58 & 148.95 \\
\hline $\mathrm{HSDX}_{\mathrm{P}_{0}} \mathrm{X} \mathrm{V}_{1}$ & $16 \times 0 \times 250$ & $142.66^{\mathrm{h}}$ & $150.00^{\mathrm{e}}$ & 113.37 & 148.45 \\
\hline $\mathrm{HSDX}_{\mathbf{P}} \mathrm{X} \mathrm{V}_{2}$ & $16 \times 0 \times 500$ & $144.66^{\mathrm{hg}}$ & $156.66^{\mathrm{cd}}$ & 113.22 & 148.64 \\
\hline $\mathrm{HSDX}_{\mathrm{P}_{1}} \mathrm{X} \mathrm{V}_{1}$ & $16 \times 1 \times 250$ & $142.66^{\mathrm{h}}$ & $153.33^{\mathrm{de}}$ & 113.25 & 148.54 \\
\hline $\mathbf{H S D X} \mathbf{P}_{1} \mathbf{X} \mathbf{V}_{2}$ & $16 \times 1 \times 500$ & $146.33^{\mathrm{fe}}$ & $158.00^{\mathrm{bc}}$ & 113.12 & 149.55 \\
\hline $\mathrm{HSDX}_{\mathbf{P}_{2}} \mathrm{X} \mathrm{V}_{1}$ & $16 \times 2 \times 250$ & $143.66^{\mathrm{hg}}$ & $155.00^{\mathrm{cd}}$ & 113.35 & 147.90 \\
\hline $\mathbf{H S D X} \mathbf{P}_{2} X \mathbf{V}_{2}$ & $16 \times 2 \times 500$ & $146.66^{\mathrm{fe}}$ & $156.33^{\mathrm{cd}}$ & 113.58 & 148.95 \\
\hline MSE & & 0.93 & 1.21 & 12.37 & 4.48 \\
\hline
\end{tabular}

Mean having similar letters in each column are not significantly different.

Plasma aspartate aminotransferase (AST) and plasma alanine aminotransferase (ALT):

Results obtained in table (5) revealed that the broiler chicks kept under LSD recorded significantly the lower averages of plasma AST and ALT than those kept under HSD at 4 and 6 wks of bird's age. This result agreed with those reported by Byung $\boldsymbol{e t}$ al (2018) who stated that plasma blood aspartate aminotransferase and alanine aminotransferase were significant higher $(\mathrm{P}<0.05)$ in the high stocking density groups than those raised in low and medium stocking density one. Chicks fed diet supplemented with probiotic at a level of $2 \mathrm{~g} / \mathrm{kg}$ diet showed significantly the lowest averages of plasma AST and ALT (19.11 and 16.11 U/L, respectively), followed by those fed diet supplemented with $1 / \mathrm{g}$ (19.15 and 16.27 , respectively U/L), compared with those fed on diet with non supplemented probiotic (21.29 and $18.54 \mathrm{U} / \mathrm{L}$, respectively) at the $6 \mathrm{wk}$ of bird's age. Concerning to the effect of V. D3 on liver function, it is observed that chicks fed diet supplemented with V.D3 at a level of $500 \mathrm{IU} / \mathrm{Kg}$ diet decreased significantly averages of plasma AST and ALT, than those fed on diet supplemented with V.D3 at a level of $250 \mathrm{IU} / \mathrm{Kg}$ diet at the $4^{\text {th }}$ and $6^{\text {th }}$ wks of bird's age. The interaction effect between LSD X P2 X V1 significantly decreased average of plasma AST. While, the interaction effect between LSD X P1 X V2 significantly decreased average of plasma ALT at 4 and 6 weeks of bird's age, compared with different interaction effects. 
Table 5. Least -square means and pooled standard error for plasma AST and ALT of different experimental groups as affected by studied factors

\begin{tabular}{|c|c|c|c|c|c|}
\hline \multirow{2}{*}{ Treatments } & \multirow{2}{*}{ level } & \multicolumn{2}{|c|}{ Plasma (AST) (U/L)at } & \multicolumn{2}{|c|}{ Plasma (ALT) (U/L)at } \\
\hline & & $4 w K s$ & 6WKs & $4 \mathrm{wKs}$ & 6WKs \\
\hline Stoking density(SD) & 10 & $15.16^{\mathrm{b}}$ & $19.55^{\mathrm{b}}$ & $11.27^{\mathrm{b}}$ & $16.70^{\mathrm{b}}$ \\
\hline bird $/ \mathrm{m}^{2}$ & 16 & $15.55^{\mathrm{a}}$ & $20.08^{\mathrm{a}}$ & $11.71^{\mathrm{a}}$ & $17.23^{\mathrm{a}}$ \\
\hline MSE & & 0.07 & 0.07 & 0.06 & 0.10 \\
\hline \multirow{3}{*}{$\begin{array}{c}\text { Probiotic (P) } \\
\mathrm{g} / \mathrm{kg}\end{array}$} & 0 & $15.94^{\mathrm{a}}$ & $21.29^{\mathrm{a}}$ & $12.08^{\mathrm{a}}$ & $18.54^{\mathrm{a}}$ \\
\hline & 1 & $15.15^{\mathrm{b}}$ & $19.15^{\mathrm{b}}$ & $11.27^{\mathrm{b}}$ & $16.27^{\mathrm{b}}$ \\
\hline & 2 & $14.98^{\mathrm{b}}$ & $19.11^{\mathrm{b}}$ & $11.12^{\mathrm{b}}$ & $16.10^{\mathrm{b}}$ \\
\hline MSE & & 0.07 & 0.09 & 0.10 & 0.12 \\
\hline \multirow{2}{*}{ Vitamin D3(V.D3)IU/kg } & 250 & $15.56^{\mathrm{a}}$ & $20.22^{\mathrm{a}}$ & $11.68^{\mathrm{a}}$ & $17.31^{\mathrm{a}}$ \\
\hline & 500 & $15.16^{\mathrm{b}}$ & $19.41^{\mathrm{b}}$ & $11.30^{\mathrm{b}}$ & $16.62^{\mathrm{b}}$ \\
\hline MSE & & $\mathbf{0 . 0 7}$ & $\mathbf{0 . 0 7}$ & 0.06 & 0.10 \\
\hline \multicolumn{6}{|l|}{ Interaction } \\
\hline $\operatorname{LSDX} P_{0} X V_{1}$ & $10 \times 0 \times 250$ & $16.16^{\mathrm{a}}$ & $21.66^{\mathrm{b}}$ & $12.36^{\mathrm{a}}$ & $19.03^{\mathrm{ab}}$ \\
\hline $\operatorname{LSDX}_{\mathrm{P}_{0}} \mathrm{X} \mathrm{V}_{2}$ & $10 \times 0 \times 500$ & $15.33^{\mathrm{dc}}$ & $20.00^{\mathrm{c}}$ & $11.43^{\mathrm{c}}$ & $17.16^{\mathrm{c}}$ \\
\hline $\operatorname{LSDX} P_{1} X V_{1}$ & $10 \times 1 \times 250$ & $15.16^{\mathrm{ce}}$ & $19.36^{\mathrm{de}}$ & $11.31^{\mathrm{cd}}$ & $16.40^{\mathrm{cd}}$ \\
\hline $\operatorname{LSDX} P_{1} X V_{2}$ & $10 \times 1 \times 500$ & $14.90^{\text {ce }}$ & $19.33^{\mathrm{de}}$ & $10.80^{\mathrm{e}}$ & $15.50^{\mathrm{e}}$ \\
\hline $\operatorname{LSDX} \mathbf{P}_{2} X V_{1}$ & $10 \times 2 \times 250$ & $14.80^{\mathrm{de}}$ & $18.79^{\mathrm{ef}}$ & $10.90^{\mathrm{de}}$ & $15.93^{\mathrm{de}}$ \\
\hline $\operatorname{LSDX} \mathbf{P}_{2} X \mathbf{V}_{2}$ & $10 \times 2 \times 500$ & $14.63^{e}$ & $19.00^{\mathrm{e}}$ & $10.83^{\mathrm{e}}$ & $16.20^{\text {de }}$ \\
\hline 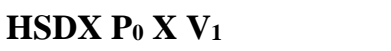 & $16 \times 0 \times 250$ & $16.33^{\mathrm{a}}$ & $22.33^{\mathrm{a}}$ & $12.50^{\mathrm{a}}$ & $19.50^{\mathrm{a}}$ \\
\hline $\mathrm{HSDX}_{\mathrm{P}_{0} \mathrm{X}} \mathrm{V}_{2}$ & $16 \times 0 \times 500$ & $15.93^{\mathrm{ab}}$ & $21.16^{\mathrm{b}}$ & $12.03^{\mathrm{b}}$ & $18.46^{\mathrm{b}}$ \\
\hline HSDX $P_{1} X V_{1}$ & $16 \times 1 \times 250$ & $15.43^{\mathrm{bc}}$ & $19.70^{\mathrm{dc}}$ & $11.53^{\mathrm{c}}$ & $16.66^{\mathrm{cd}}$ \\
\hline $\mathrm{HSDX}_{\mathrm{P}_{1}} \mathrm{X} \mathrm{V}_{2}$ & $16 \times 1 \times 500$ & $15.13^{\text {ce }}$ & $18.80^{\mathrm{ef}}$ & $11.43^{\mathrm{c}}$ & $15.83^{\mathrm{de}}$ \\
\hline $\mathrm{HSDX}_{\mathrm{P}_{2}} \mathrm{X} \mathrm{V}_{1}$ & $16 \times 2 \times 250$ & $15.64^{\mathrm{e}}$ & $19.33^{\mathrm{de}}$ & $11.46^{\mathrm{c}}$ & $16.36^{\mathrm{cd}}$ \\
\hline $\mathrm{HSDX}_{\mathrm{P}_{2}} \mathrm{X} \mathrm{V}_{2}$ & $16 \times 2 \times 500$ & $15.05^{\mathrm{ce}}$ & $19.20^{\mathrm{de}}$ & $11.30^{\mathrm{cd}}$ & $16.60^{\mathrm{cd}}$ \\
\hline MSE & & 0.17 & 0.19 & 0.14 & 0.25 \\
\hline
\end{tabular}

Mean having similar letters in each column are not significantly different.

\section{Plasma uric acid and creatinine}

Data presented in table (7) revealed that, no significant variations were found in plasma uric acid and creatinine of broiler chickens due to the effect of stoking density (SD), probiotic (P), Vit.D3 (V.D3) supplementation and the interaction between them at all time of estimations. Although there were no significant effects of studied factors on kidney function, broiler chicks kept under HSD showed the lowest average of plasma uric acid (4.75 and 4.50 $\mathrm{mg} / \mathrm{dI}$ at 4 and 6 weeks of age, respectively ). However chicks kept under LSD decreased plasma creatinine $(0.22 \mathrm{mg} / \mathrm{dI})$ at 6 weeks of age $)$. Concerning to the effect of Probiotic on plasma uric acid and creatinine, it is clearly observed that chicks fed diet supplemented with probiotic at a level of 1 $\mathrm{g} / \mathrm{kg}$ diet showed lowest plasma uric acid at the 6 week of age $(4.68 \mathrm{mg} / \mathrm{dI})$, However chicks fed diet supplemented with $2 \mathrm{~g} / \mathrm{kg}$ showed the lowest plasma creatinine $(0.14 \mathrm{mg} / \mathrm{dl})$ at the 6 week of age. These result disagree with those reported by $\mathbf{L i}$ et al. (2008) who demonstrated that, there was a decrease in blood uric acid by $22.39 \%$ due to probiotic supplementation (Bacillus subtilis) of quail birds. Chicks fed diet supplemented with V.D3 at a level of
$500 \mathrm{IU} / \mathrm{Kg}$ diet showed the lowest averages of plasma uric acid (4.73 and $4.64 \mathrm{mg} / \mathrm{dI}$ at 4 and 6 weeks of age, respectively) while, chicks fed diet supplemented with V.D3 at a level of $250 \mathrm{IU} / \mathrm{kg}$ showed the lowest averages of plasma creatinine $(0.22$ and $0.19 \mathrm{IU} / \mathrm{kg}$ at 4 and 6 weeks of age, respectively). The interaction effects between LSD X P2 XV1 and between LSD X P1X V2 decreased plasma creatinine and uric acid, respectively at the 6 weeks of age when compared with different interaction applied. 
Table 7. Least - square means and pooled standard error for plasma uric acid and creatinine of different experimental groups as affected by studied factors

\begin{tabular}{|c|c|c|c|c|c|}
\hline \multirow{2}{*}{ Treatments } & \multirow{2}{*}{ level } & \multicolumn{2}{|c|}{$\begin{array}{c}\text { Plasma creatinine } \\
(\mathrm{mg} / \mathrm{dL}) \text { at }\end{array}$} & \multicolumn{2}{|c|}{ Plasma uric acid(mg/dl) at } \\
\hline & & 4WKS & 6WKS & 4WKS & 6WKS \\
\hline Stoking density (SD) & 10 & 0.25 & 0.22 & 4.77 & 4.73 \\
\hline bird $/ \mathbf{m}^{2}$ & 16 & 0.17 & 0.39 & 4.75 & 4.50 \\
\hline \multirow{2}{*}{ MSE } & & 0.017 & 0.018 & 0.53 & 0.53 \\
\hline & 0 & 0.10 & 0.14 & 4.79 & 4.70 \\
\hline \multirow[t]{2}{*}{ Probiotic $(P) \mathrm{g} / \mathrm{kg}$} & 1 & 0.09 & 0.15 & 4.85 & 4.68 \\
\hline & 2 & 0.10 & 0.14 & 4.63 & 4.71 \\
\hline MSE & & 0.021 & 0.022 & 0.65 & 0.44 \\
\hline \multirow{2}{*}{ Vitamin D3 (V.D3 ) IU/kg } & 250 & 0.22 & 0.19 & 4.78 & 4.67 \\
\hline & 500 & 0.24 & 0.30 & 4.73 & 4.64 \\
\hline MSE & & 0.018 & 0.017 & $\mathbf{0 . 5 3}$ & 0.36 \\
\hline \multicolumn{6}{|l|}{ Interaction } \\
\hline $\operatorname{LSDX}_{\mathbf{0}} \mathrm{X} \mathrm{V}_{1}$ & $10 \times 0 \times 250$ & 0.25 & 0.37 & 4.86 & 4.89 \\
\hline $\operatorname{LSDX}_{\mathbf{P}} \mathrm{X} \mathrm{V}_{2}$ & $10 \times 0 \times 500$ & 0.24 & 0.24 & 4.78 & 4.68 \\
\hline $\operatorname{LSDX}_{\mathrm{P}_{1}} \mathbf{X} \mathbf{V}_{1}$ & $10 \times 1 \times 250$ & 0.28 & 0.27 & 4.89 & 4.71 \\
\hline $\operatorname{LSDX} \mathrm{P}_{1} \times \mathrm{V}_{2}$ & $10 \times 1 \times 500$ & 0.20 & 0.26 & 4.78 & 4.45 \\
\hline $\operatorname{LSDX}_{2} \mathrm{P}_{2} \mathrm{~V}_{1}$ & $10 \times 2 \times 250$ & 0.22 & 0.15 & 4.67 & 4.76 \\
\hline $\operatorname{LSDX} \mathbf{P}_{2} \times \mathbf{V}_{2}$ & $10 \times 2 \times 500$ & 0.34 & 0.42 & 4.40 & 4.78 \\
\hline $\mathrm{HSDX}_{\mathrm{P}_{0}} \mathrm{X} \mathrm{V}_{1}$ & $16 \times 0 \times 250$ & 0.20 & 0.22 & 4.87 & 4.53 \\
\hline $\mathrm{HSDX}_{\mathbf{0}} \mathrm{X} \mathrm{V}_{2}$ & $16 \times 0 \times 500$ & 0.27 & 0.16 & 4.79 & 4.63 \\
\hline HSDX $P_{1}$ X V 1 & $16 \times 1 \times 250$ & 0.46 & 0.18 & 4.86 & 4.57 \\
\hline HSDX $P_{1} X V_{2}$ & $16 \times 1 \times 500$ & 0.17 & 0.27 & 4.83 & 4.61 \\
\hline $\mathrm{HSDX}_{2} \mathrm{P}_{2} \mathrm{~V}_{1}$ & $16 \times 2 \times 250$ & 0.32 & 0.29 & 4.40 & 4.51 \\
\hline $\mathrm{HSDX} \mathrm{P}_{2} \mathrm{X} \mathrm{V}_{2}$ & $16 \times 2 \times 500$ & 0.25 & 0.26 & 4.63 & 4.67 \\
\hline MSE & & 0.043 & 0.046 & 1.53 & 0.92 \\
\hline
\end{tabular}

Mean having similar letters in each column are not significantly different.

Plasma calcium and inorganic phosphorus:

Results obtained in table (8) showed highly significant variations in plasma calcium and inorganic phosphorus at 4 and 6 weeks of age due to the effect of Vit.D3 supplemented level and the interaction effects between all factors studied showed highly significant variations on plasma calcium and inorganic phosphorus at 4 and 6 weeks of bird's age. Broiler chicks fed on diet supplemented with V.D3 at a level of $500 \mathrm{IU} / \mathrm{Kg}$ diet showed significantly the higher averages of plasma calcium and inorganic phosphorus at 4 and 6 weeks of birds age than those fed on diet supplemented with V.D3 at a level of $250 \mathrm{IU} / \mathrm{Kg}$. Chicks fed diet supplemented with probiotic at a level of $1 \mathrm{~g} / \mathrm{kg}$ diet showed the highest averages of plasma calcium and inorganic phosphorus (10.57 and $6.57 \mathrm{mg} / \mathrm{dI}$, respectively) at the 6 week of age. The interaction between HSD X P0 X V2 and between LSD X P1 X V2 showed significantly the highest average of plasma calcium $(13.96 \mathrm{mg} / \mathrm{dl})$ and inorganic phosphorus $(7.90 \mathrm{mg} / \mathrm{dl}$ ) at the end of the experimental period compared with different interaction applied. These results are agree with those reported by Whitehead (2004) who sated that plasma blood ionized calcium, adjusted to $\mathrm{pH} 7.4$, showed increases $(\mathrm{P}<0.05)$ with increasing vitamin D3 supplementation for all combinations of $\mathrm{Ca}$ and $\mathrm{P}$ except, the diet containing $13 \mathrm{~g} \mathrm{Ca} / 5 \mathrm{~g} \mathrm{P}$. However, these results disagreed with those found by Felix Shih-Hsiang Hsiao1 (2018) who reported that, no significant effect on serum calcium and serum phosphorus of broiler chickens dietary supplementation of vitamin D3. 
Table 8. Least -square means and pooled standard error for plasma calcium and inorganic phosphorus of different experimental groups as affected by studied factors

\begin{tabular}{|c|c|c|c|c|c|}
\hline \multirow{2}{*}{ Treatments } & \multirow{2}{*}{ level } & \multicolumn{2}{|c|}{$\begin{array}{c}\text { Plasma calcium } \\
(\mathrm{mg} / \mathrm{dI}) \text { at }\end{array}$} & \multicolumn{2}{|c|}{$\begin{array}{c}\text { Plasma inorganic } \\
\text { phosphorus }(\mathrm{mg} / \mathrm{dL}) \text { at }\end{array}$} \\
\hline & & $4 \mathrm{wKS}$ & 6WKS & $4 w K S$ & 6WKS \\
\hline Stoking density (SD) & 10 & 12.12 & 10.57 & 8.20 & 6.54 \\
\hline bird $/ \mathbf{m}^{2}$ & 16 & 12.14 & 10.41 & 7.95 & 6.45 \\
\hline MSE & & 0.22 & 0.16 & 0.15 & 0.10 \\
\hline \multirow{3}{*}{$\begin{array}{c}\text { Probiotic (P) } \\
\text { g } / \mathrm{kg}\end{array}$} & 0 & 12.19 & 10.36 & 7.98 & 6.54 \\
\hline & 1 & 12.12 & 10.57 & 7.99 & 6.57 \\
\hline & 2 & 12.08 & 10.54 & 8.25 & 6.37 \\
\hline MSE & & 0.26 & 0.19 & 0.19 & 0.13 \\
\hline \multirow{2}{*}{ Vitamin D3 (V.D3)IU/kg } & 250 & $10.81^{\mathrm{b}}$ & $9.24^{\mathrm{b}}$ & $7.35^{\mathrm{b}}$ & $5.43^{\mathrm{b}}$ \\
\hline & 500 & $13.45^{\mathrm{a}}$ & $11.74^{\mathrm{a}}$ & $8.80^{\mathrm{a}}$ & $7.55^{\mathrm{a}}$ \\
\hline MSE & & 0.22 & 0.16 & 0.15 & 0.10 \\
\hline \multicolumn{6}{|l|}{ Interaction } \\
\hline $\operatorname{LSDX}_{\mathrm{P}_{0}} \mathrm{X} \mathrm{V}_{\mathbf{1}}$ & $10 \times 0 \times 250$ & $9.43^{\mathrm{b}}$ & $10.70^{\mathrm{b}}$ & $7.60^{\mathrm{bc}}$ & $5.53^{\mathrm{b}}$ \\
\hline LSDX $\mathbf{P}_{0} \mathrm{X} \mathrm{V}_{2}$ & $10 \times 0 \times 500$ & $11.36^{\mathrm{a}}$ & $13.26^{\mathrm{a}}$ & $8.90^{\mathrm{a}}$ & $7.50^{\mathrm{a}}$ \\
\hline $\operatorname{LSDX} \mathbf{P}_{1} X \mathrm{~V}_{1}$ & $10 \times 1 \times 250$ & $9.70^{\mathrm{b}}$ & $10.76^{\mathrm{b}}$ & $7.20^{c}$ & $5.60^{\mathrm{b}}$ \\
\hline $\operatorname{LSDX} \mathbf{P}_{1} X \mathbf{V}_{2}$ & $10 \times 1 \times 500$ & $11.70^{\mathrm{a}}$ & $13.36^{\mathrm{a}}$ & $8.96^{\mathrm{a}}$ & $7.90^{\mathrm{a}}$ \\
\hline $\operatorname{LSDX} \mathbf{P}_{2} X \mathrm{~V}_{1}$ & $10 \times 2 \times 250$ & $9.33^{b}$ & $10.93^{\mathrm{b}}$ & $7.65^{\mathrm{bc}}$ & $5.10^{\mathrm{b}}$ \\
\hline $\operatorname{LSDX}_{\mathrm{P}_{2}} \mathrm{X} \mathrm{V}_{2}$ & $10 \times 2 \times 500$ & $11.90^{\mathrm{a}}$ & $13.90^{\mathrm{a}}$ & $9.00^{\mathrm{a}}$ & $7.63^{\mathrm{a}}$ \\
\hline 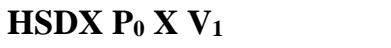 & $16 \times 0 \times 250$ & $8.60^{\mathrm{b}}$ & $10.83^{\mathrm{b}}$ & $7.13^{c}$ & $5.43^{\mathrm{b}}$ \\
\hline $\mathrm{HSDX}_{\mathbf{P}} \mathrm{X} \mathrm{V}_{2}$ & $16 \times 0 \times 500$ & $12.06^{\mathrm{a}}$ & $13.96^{\mathrm{a}}$ & $8.30^{\mathrm{abc}}$ & $7.70^{\mathrm{a}}$ \\
\hline 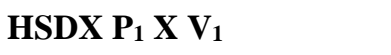 & $16 \times 1 \times 250$ & $9.16^{\mathrm{b}}$ & $11.36^{\mathrm{b}}$ & $7.10^{\mathrm{c}}$ & $5.50^{\mathrm{b}}$ \\
\hline HSDX $P_{1} X V_{2}$ & $16 \times 1 \times 500$ & $11.76^{\mathrm{a}}$ & $13.00^{\mathrm{b}}$ & $8.70^{\mathrm{ab}}$ & $7.30^{\mathrm{a}}$ \\
\hline 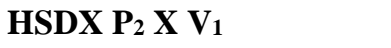 & $16 \times 2 \times 250$ & $9.16^{\mathrm{b}}$ & $10.30^{\mathrm{a}}$ & $7.50^{\mathrm{bc}}$ & $5.64^{b}$ \\
\hline $\mathrm{HSDX}_{\mathbf{P}} \mathrm{X} \mathrm{V}_{2}$ & $16 \times 2 \times 500$ & $11.76^{\mathrm{a}}$ & $13.40^{\mathrm{b}}$ & $8.96^{\mathrm{a}}$ & $7.30^{\mathrm{a}}$ \\
\hline MSE & & 0.33 & 0.54 & 0.40 & 0.26 \\
\hline
\end{tabular}

Mean having similar letters in each column are not significantly different

\section{References}

Adeyemo, G. O.; Fashola, O. O.; Ademulegun, T. I. (2016).Effect of Stocking Density on the Performance, Carcass Yield and Meat Composition of Broiler Chickens. BBJ, 14 (1): 17.

Almeida Paz, I.C.L; Mendes, A.A.; Balog, A.; Vulcano, L.C; Ballarin, A.W; Takahashi, S.E, Komyiama, C.M.; Silva, M.C. ; Cardoso, K.F.G. (2008). Study on the Bone Mineral Density of the Femur of Broilers Suffering Femur Degeneration. Brazilian Journal of Poultry Science;10(2):103-108.

Byung-Sung, P.; Kyung-Hwan, U.; Sang, O. P. and Victor, A. Z. (2018).Effect of stocking density on behavioral traits, blood biochemical parameters and immune responses in meat ducks exposed to heat stress. Arch. Anim. Breed., 61, 425-432.

Dozier, W. A.; Thaxton, J. P. ; Purswell, J. L. ; Olanrewaju, H. A. ; Branton, S. L. and Roush, W. B. (2006). Stocking density effects on male broilers grown to 1.8 kilograms of body weight. Poult. Sci.85:344-351.
Duncan, M. B. (1955): Multiple ranges and multiple F-test. Biometrics, 11: 1-42.

Estevez, I.( 2007). Density allowances for broilers: Where to set thlimits? Poult. Sci. 86:12651272.

Feddes, J. J. R.; Emmanuel, E. J. and Zuidhof M. J. (2002). Broiler performance, bodyweight variance, feed and water intake, and carcass quality at different stocking densities. Poult. Sci. 81:774-779.

Felix Shih-Hsiang Hsiao1, Yeong-Hsiang Cheng2, Jin-Cheng Han3, Ming-Huang Chang4, YuHsiang Yu2* ., 2018 Effect of different vitamin D3 metabolites on intestinal calcium homeostasis related gene expression in broiler chickens. R. Bras. Zootec., 47:e20170015, 2018.

Fraser, D and Kodicek, E. (1970).Unique biosynthesis by kidney of a biologically active vitamin D3 metabolite.Nature228,764-766. doi:10.1038/228764a.

Goo D.; Kim, J. H. ; Choi, H. S. ; Park, G. H. ; Han, G. P. and Kil1, D. Y. (2019). Effect of stocking density and sex on growth performance, meat quality, and intestinal barrier function in broiler chickens. 2019 Poultry Science 98:11531160. 
Guardia.S ; B. Konsak , S. Combes ; Levenez, F.; Cauquil , L. ; Guillot , J.-F. ; MoreauVauzelle, C. ; Lessire, M. ; Juin, H. and Gabriel, I. (2011). Effects of stocking density on the growth performance and digestive

Huang, M. K.; Choi, Y. J.; Houde, R. ; Lee, J. W. and Zhao, X. (2004). Effects of Lactobacilli and an Acidophilus fungus on the production performance and immune responses in broiler chickens. Poult. Sci. 83:788-795.

Hurwitz, S.; Fishman, S.; Bar, A. and Talpaz, H. (1984). Role of the 1, 25 dihydroxycholecalciferol-regulated component of calcium absorption in calcium homeostasis. Progress in Clinical and Biological Research 168, 357-362.

Ignatova, M.; Sredkova, V. and Marasheva, V. (2009). Effect of dietaryinclusion of probiotic on chickens performance and some bloodindices. Biotechnol. Anim. Husband. 25: 1079-1085.

Li, L. L.; Hou, Z. P.; Li, T. J. ; Wu, G. Y. ; Huang, R. L. ; Tang, Z. R. ; Yang, C. B. ; Gong, J. ; Yu, H. and Kong, X. F. (2008). Effects of dietary probiotic supplementation on ileal digestibility of nutrients and growth performance in 1 to 42 -day-old broilers. J. Sci. Food Agric.88:3542.

Lymboussaki, A.; Gemelli, C.; Testa, A.; Facchini, G.; Ferrari, F.; Mavilio, F. and Grande, A. PPAR (2009)delta is a ligand-dependent negative regulator of vitamin D3-induced monocyte differentiation. Carcinogenesis, 30: 230-237.

Mohammadi Gheisar. M; Hosseindoust, A. ; Kim, I.H. (2016). Effects of dietary Enterococcus faecium on growth performance, carcass characteristics, faecal microbiota, and blood profile in broilers. Veterinarni Medicina, 61, 2016 (1): $28-34$

Mountzouris, K. C.; Balaskas, C.; Xanthakos, I. ; Tzivinikou, A. and Fegeros, K. (2009). Effects of a multi-species probiotic on biomarkers of competitive exclusion efficacy in broilers challenged with Salmonella enteritidis. Br. Poult. Sci. 50:467-478.

NRC. (1994). Nutrient Requirements of Poultry. 9th rev. ed. Natl. Acad. Press, Washington, DC.

Puron, D.; Santamaria, R.; Segaura, J. C. and Alamilla, J. L. (1995). Broiler performance at different stocking densities. J. Appl. Poult.Res. 4:55-60.
RudzaniSiaga, Joseph JimuBaloyi, Mashudu Daniel Rambau and KowBenyi (2017). Effects of Stocking Density and Genotype on the Growth Performance of Male and Female Broiler Chickens.Asian J. Poult. Sci., 11 (2): 96-104, 2017

SAS Institute (2004): SAS User's Guide. Release 8.2.Ed. SAS Institute Inc. Cary. NC.

Sherif, K. h. El. (2009b).Effect of using probiotics and enzymes with plant-protein diets in broiler performance. J. Agric. Sci., Mansoura Univ., 34 (5): 4493-4505.

Sohail, M. U.; Hume, M. E. ; Byrd, J. A. ; Nisbet, D. J. ; Ijaz, A. ; Sohail, A.; Shabbir, M. Z. and Rehman, H. (2012). Effect of supplementation of prebiotic mannan-oligosaccharides and prebiotic mixture on growth performance of broilers subjected to chronic heat stress. Poult. Sci. 91:2235-2240.

Thaxton, J. P.; Dozier III, W. A.; Branton, S. L. ; Morgan, G. W. ; Miles, D. W. ; Roush, W. B. Lott, B. D. and Vizzier-Thaxton, Y. (2006). Stocking density and physiological adaptive responses of broilers. Poult. Sci. 85:819-824.

Teo, A. Y.; and Tan, H. M. (2007). Evaluation of the performance and intestinal gut microflora of broilers fed on corn-soy diets supplemented with Bacillus subtilis PB6 (CloSTAT). J. Appl. Poult. Res. 16:296-303.

Tong, H. B.; Lu, J.; Zou, J. M.; Wang, Q. and Shi, S. R. (2012). Effects of stocking density on growth performance, carcass yield, and immune status of a local chicken breed. Poult. Sci. 91:667-673.

Waldenstedt, L. (2006). Nutritional factors of importance for optimal leg health in broilers: A review. Animal Feed Science and Technology 2006;126(3):291-307.

Whitehead, C. C.; McCormack, H. A. ; McTeir, L. and Fleming, R. H. (2004) . High vitamin D3 requirements in broilers for bone quality and prevention of tibia dyschondroplasia and interactions with dietary calcium, available phosphorus and vitamin A. Br. Poult. Sci. 45:425-436.

Yu, B.; Liu, J. R. ; Hsiao, F. S. and Chiou, P. W. S. (2008). Evaluation of Lactobacillus reuteri Pg4 strain expressing heterologous $\beta$-glucanase as a prebiotic in poultry diets based on barley. Anim. Feed Sci. Technol. 141:82-91. 